\title{
Para Ovarian Benign Multicystic Mesothelioma-A Case Report
}

\author{
Wills G Sheelaa ${ }^{1 *}$ and A Gowri Bai ${ }^{2}$ \\ ${ }^{1}$ Associate Professor, Department of Obstetrics and Gynaecology, India \\ ${ }^{2}$ Professor, Department of Obstetrics and Gynaecology, India
}

Received: October 14, 2017; Published: October 23, 2017

*Corresponding author: Dr. Wills G Sheelaa, Associate Professor, Department of Obstetrics and Gynaecology, SMVMCH, Puducherry; Flat no: H3, Sand stone, Anurag garden, Tholkapiyar street, New Perungalaur, Chennai-600063 India; Email: drgwillssheelaa@yahoo.in

\begin{abstract}
Mesotheliomas are mesenchymal neoplasms which originate in the lining membrane of various serous cavities and peritoneum. These are benign tumours slow growing. They are incidentally found during laparotomies for ovarian tumours with ascitis. Common in women, exposed to asbestos. We present a rare para ovarian benign multi cystic mesothelioma in a 41-years-old woman who presented like malignant ovarian tumour, with a huge cyst of size $25 \times 15 \mathrm{~cm}$ containing four liters of haemorrhagic fluid and four small cysts of size $6 \times 4 \mathrm{~cm}$. Histological picture was confirmative for para ovarian benign mesothelioma.
\end{abstract}

Keywords : Mesothelioma; Benign Ovarian Tumour; Multiloculated Cyst

\section{Introduction}

The peritoneum is a mesothelial lining of the abdominal cavity and intra parietal viscera. Mesotheliomas are mesenchymal neoplasms which originate in the lining membrane of various serous cavities, pleural, pericardial and peritoneum. Women present with distension of abdomen, pain, ascites and palpable mass. Most are incidental surgical findings accompanied by multiple cystic peritoneal lesions. Histological picture is predominantly papillary with differentiated mesothelial cells. Often differentiated with adenomatoid tumours and tumours of ovarian epithelium. Peritoneal mesothelioma is a form of mesothelioma that affects the lining of the abdomen often misdiagnosed as ovarian cancer. It is common in asbestos exposed woman. These are slowly progressive. Surgical removal is the treatment. Has high rate or recurrence treated with ant estrogens. A case of para ovarian benign multicystic mesothelioma, who presented as malignant ovarian tumour is presented for its rarity.

\section{Case Report}

A 41-years- old $\mathrm{P}_{2} \mathrm{~L}_{2}$ sterilized woman reported to the Gynaecology OPD of Sri Manakula Vinayagar Medical College and Hospital, Puducherry, India with the complaint of distension of abdomen, loss of appetite and pain abdomen for three months with regular periods. There was no history of exposure to asbestos. On examination there was a cystic mass of size $34 \times 32 \mathrm{~cm}$, occupying the entire abdomen with side to side movement. There was no ascites. Uterus was retroverted and normal in size. Right fornix was occupied by a cystic mass which was extending up to the umbilicus. Tumour marker CA125 was elevated. Ultrasound scan and Computed tomography scan revealed a large cystic mass occupying lower abdomen. Largest cyst was $25 \mathrm{~cm}$ x $15 \mathrm{~cm}$ with multiple thick septation within the cyst. Multiple small cysts of size $6 \times 4 \mathrm{~cm}$ were also seen on right ovary. A diagnosis of malignant right ovarian tumour was made. The patient underwent staging laparotomy. Uterus was normal in size. Left tube and ovary were normal. Right side-tube had fimbrial cyst $5 \times 4 \mathrm{~cm}$ with haemorrhagic fluid. A multiloculated right ovarian cyst, largest measuring $25 \times 15 \mathrm{~cm}$ filled with haemorrhagic fluid about four liters, and four smaller cysts of size $6 \mathrm{cmx} 4 \mathrm{~cm}$ were present. Same removed with ovary. There was no papillary projection or solid areas. Minimal clear peritoneal fluid staging was 0 as tumour was benign. Uterus cervix and right diseased ovary with multilocilated cysts and the normal left ovary was also removed with tubes, abdomen explored, left ovary was removed because we don't have any facility for frozen section of the normal ovary. Omental biopsy taken and abdomen closed in layers. Uterus cervix both tubes left normal ovary right ovary with multiple cysts were send for histopathalogical examination. HPE REPORT - a diagnosis of Para ovarian multi cystic mesothelioma was made. The Omentum also showed mesothelial proliferation. Her postoperative period was uneventful and the patient was discharged on the $10^{\text {th }}$ day patients was followed after 3 months, 6months and 1 year, patient is healthy and leading a normal life. 


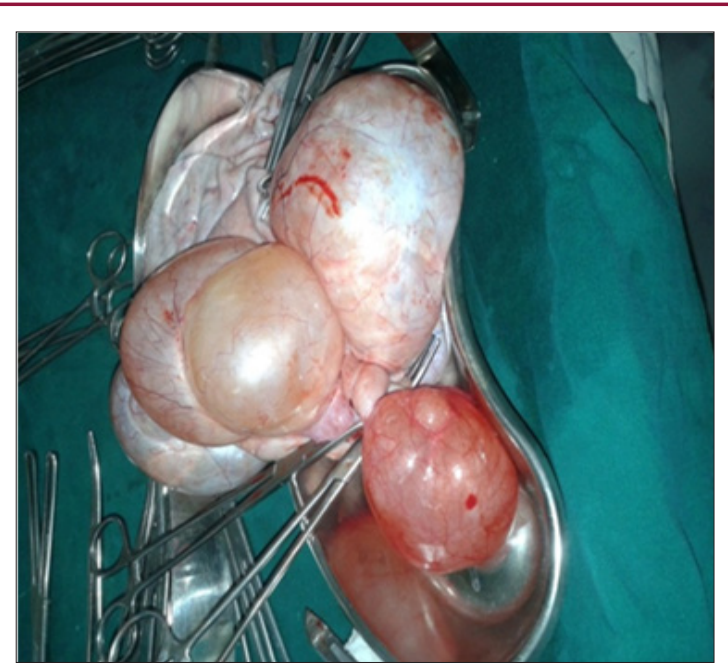

Figure 1: Post operative specimen showing of right ovary with massive ovarian multiple cysts which contained 4 liters of haemorrhagic fluid.

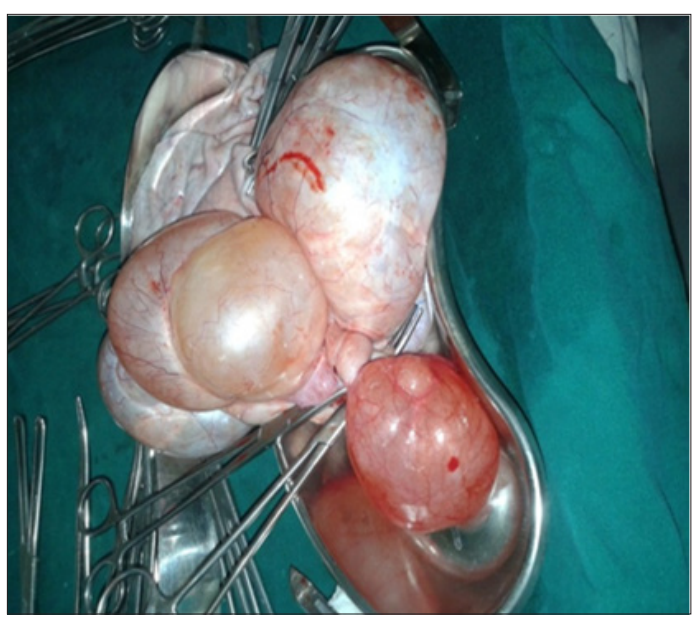

Figure 2 : Four small cysts of size $6 \times 4 \mathrm{~cm}$.

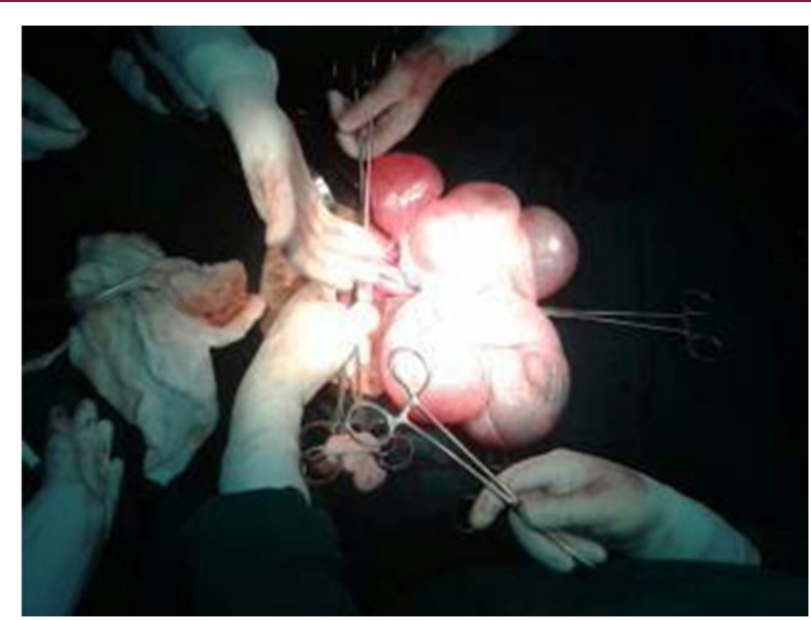

Figure 3 : Intra operative picture of pedical of the cysts with right ovarian tissue

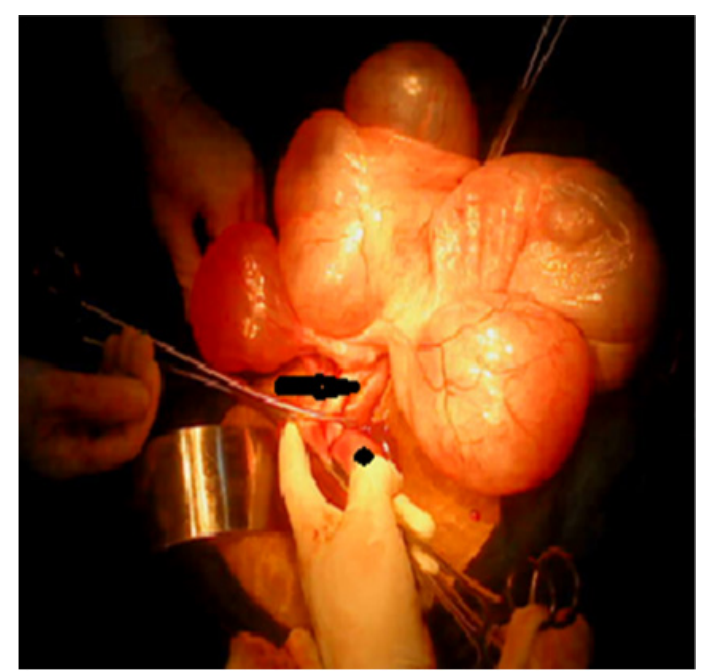

Figure 4 : The uterus (dot shows the uterus and arrow shows right ovarian pedicle) along with multiple ovarian cysts.

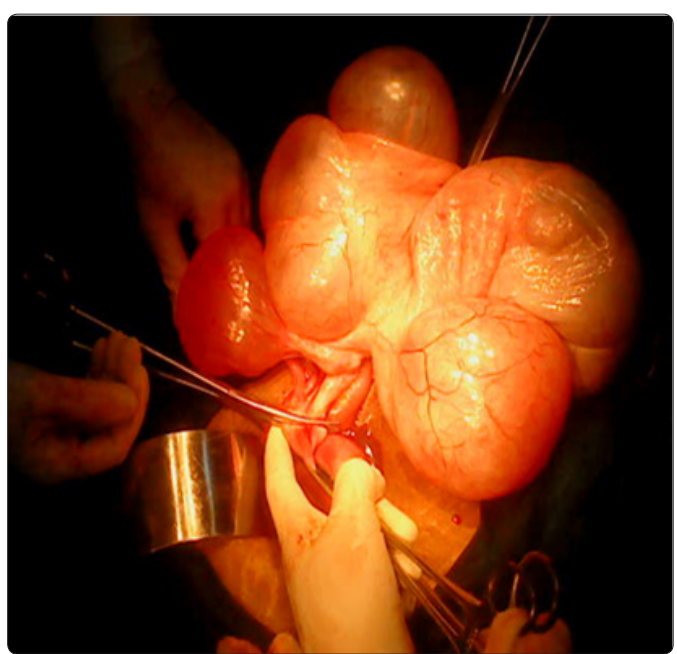

Figure 5

a. Gross Description of Specimen: The uterus, cervix, left fallopian tube and left ovary was normal. Right fallopian tube and ovary consisted of a cyst of size $35 \mathrm{~cm}$ x $30 \mathrm{~cm}$ attached to ovary. External surface of ovary was bosselated and congested. Cut section showed multiloculated cyst filled with serous fluid. Cysts walls were thin, one focus showing tiny papillary excretion. No solid areas were seen. Ovary was attached to one of the locules measuring $5 \times 4 \times 2 \mathrm{~cm}$. Cut section showed tiny cortical cyst (Figures 1-5).

b. Microscopic Description: Cyst was lined by flattened to pseudo-stratified layer of benign mesothelial cells with focal papillary projections into the lumen. Papillae showed central fibrovascular core and single layer of mesothelial lining. Cyst wall was thin and fibro collagenous. Right ovary showed follicular cyst and resolving corpus luteum. After histological evaluation, a diagnosis of Para ovarian multi cystic mesothelioma was made. The Omentum also showed mesothelial proliferation. 


\section{Discussion}

Papillary mesothelioma of ovary is rare benign tumours. Often, it is an incidental surgical finding when laparatomy is done for ovarian epithelial tumours, with histological pattern of predominantly papillary well differentiated mesothelial cells, and prominent stromal infiltrate [1]. Benign multicystic peritoneal mesothelioma (BMPM) [2]. These tumours are rare and occur mainly in women in their reproductive age associated with previous surgery. These tumours are slowly progressive. Patients present with diffuse abdominal pain, nausea and vomiting, painful mass in the upper abdomen. Big cystic mass of ovary with small cysts containing clear fluid is the usual intra operative finding [3]. Imaging techniques like ultrasonography, computerized tomography and magnetic resonance image can demonstrate the lesion. Surgery is the only effective treatment. Complete removal of the cystic lesion should be aimed at to avoid local recurrence. Recurrence occurs frequently and is treated with hormonal therapy with antiestrogens or gonadotropin releasing analogous [3].

Cases of malignant mesothelioma of the peritoneum have been reported by authors Pontone P et al. [4] from Italy. Peken T [5] from Turkey has reported a case of malignant epithelial peritoneal mesothelioma in pregnancy. Reid [6] has reported that some cases of ovarian cancer in women with history of asbestos exposure are misdiagnosed as peritoneal mesothelioma. In our study there is no exposure to any asbestos. We report a rare case of a 41 year old sterilized woman, who presented like malignant ovarian tumour, with a surprise postoperative histological diagnosis of paraovarian, benign multicystic mesothelioma.

\section{Conclusion}

Para ovarian benign multicystic mesotheliomas are rare tumours. Only few cases have been reported. Malignant mesothelioma of the peritoneum is more common. Surgery is the first line of treatment. Recurrence of the tumour is common and is treated by ant estrogens and gonadotropin releasing analogous. Often these tumours mimic ovarian malignant tumours and are diagnosed incidentally during laparatomy. Histological diagnosis is confirmative.

\section{References}

1. Addis BJ, Fox H (1989) Papillary mesothelioma of ovary. Histopathology $7(2): 287-298$.

2. Safioleas MC, Constatntinos K, Michael S, Konstantinos G, Constantinos S, et al. (2006) Benign multicystic peritoneal mesothelioma: A case report and review of the literature. World J 12(35): 5739-5742.

3. Michael C Safioleas, Kontzoglou Constantinos, Stamatakos Michael, Safioleas Constantinos, et al. (2006) Benign multicystic peritoneal mesothelioma: A case report and review of the literature.

4. Pontone P, Crescenzi A, el Osta K, Petruzziello L, Petruzziello F, de Anna $\mathrm{L}$, et al. (1992) (Article in Italian) Malignant peritoneal mesothelioma : apropos a new case. Ann Ital Chir 63(6): 807-810.

5. Pekin T, Cosar E, Dedeoglu N, Cakalagaoglu F, Gokaslan H, et al. (2004) Diffuse malignant epithelial peritoneal mesothelioma in pregnancy. A case report and literature review. Eur J Gynaecol Oncol 25(1): 119-122.

6. Reid A, de Klerk N, Musk AW (2011) Does exposure to asbestos cause ovarian cancer? A systematic literature review and meta-analysis. Cancer Epidemiol Biomarkers Prev 20(7): 1287-1295.

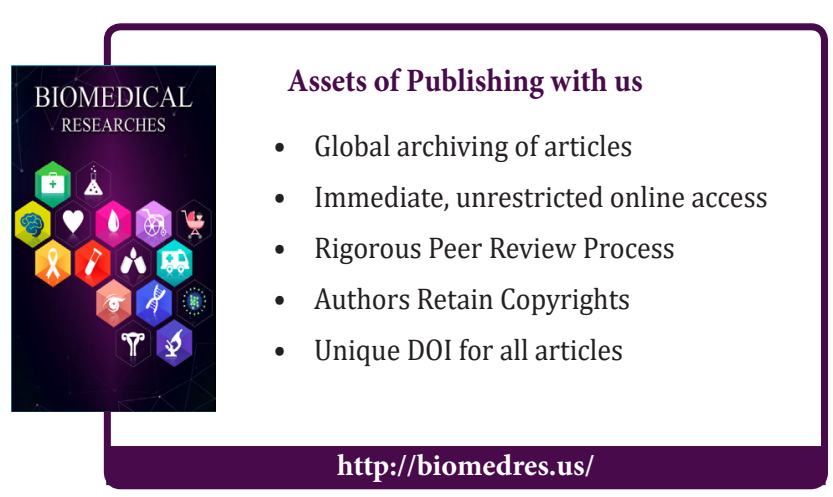

